\title{
(DES)TEJIENDO EL ENTRAMADO DE RELACIONES SOCIALES EN LA EMILIA. UNA APROXIMACIÓN A LA CONSTRUCCIÓN SOCIAL DEL PAISAJE
}

\author{
Daniela Cadenas ${ }^{1}$
}

Fecha de defensa: 9 de octubre de 2019

Director: Dr. Juan Bautista Leoni

Jurados: Lic. Diana Tamburini y Lic. Mariana Algrain

Palabras clave: fábrica, pueblo, paisaje, poder

\section{Introducción}

Los paisajes, entendidos como espacios subjetivos, se encuentran significativamente constituidos. Las formas que adquieren y las transformaciones que experimentan responden a un complejo entramado conformado por diversos procesos sociohistóricos que, a su vez, contribuyen a reproducir. Sus elementos, en tanto parte de un orden material históricamente conformado, emergen en el presente como marcas materiales a partir de las cuales comprender las relaciones sociales que les dieron forma.

La expansión del capitalismo industrial hacia fines del siglo XIX, junto con un estilo moderno que pretendía alcanzar todas las esferas de la vida cotidiana, incluyendo la organización y diseño del espacio, constituye un ejemplo sobre cómo las espacialidades representan $\mathrm{y}$, a su vez, reproducen un orden social instaurado desde las estructuras de poder (Acuto, 1999; Foucault, 2000[1982]; Foucault, 2014[1975]; McGuire, 1991; Tilley, 1994). Así, el paisaje de la fábrica-pueblo La Emilia resultó apropiado para analizar la espacialidad como producto/ productora de un determinado orden social que entrelaza sujetos, objetos, lugares, sentidos y discursos, conformando una red de relaciones en la que se configura la vida cotidiana de quienes lo habitaron y habitan.

La Emilia fue una empresa textil fundada por inmigrantes españoles en 1892 a orillas del Arroyo del Medio, en el Partido de San Nicolás (Buenos Aires), en torno a la que se conformó una comunidad dentro de un predio privado, propiedad de la compañía. El concepto fabrica-pueblo, derivado del término en inglés company-town, refiere a establecimientos en los que una sola compañía se constituye como la única fuente de trabajo y a la que, además, prácticamente todas las instalaciones (viviendas, negocios, escuelas, espacios recreativos) se encuentran subordinadas. En este sentido, el concepto resultó apropiado en tanto el caso de estudio no puede entenderse sólo como una fábrica por un lado, ni como un pueblo por otro, sino a partir de los vínculos que los entrelazan y de las particularidades que de ello surgen.

De esta historia entrelazada, emerge el discurso tradicional de "gran familia", que supone relaciones sociales carentes de conflictos, y un vínculo armonioso y familiar entre dueños y obreros. Resultó crucial, entonces, buscar nuevas perspectivas a partir de las cuales observar el paisaje e intentar penetrar esa visión hegemónica que no deja lugar para conflictos y contradicciones y que merece, por tanto, ser problematizada al partir del supuesto de que en la construcción social del paisaje tienen lugar relaciones asimétricas de poder, intereses contrapuestos y disputas por el control social.

\section{Objetivos}

El objetivo general de esta tesis consistió en el análisis de la construcción social del paisaje de La Emilia, por medio del diseño urbano en sus aspectos físicos y sociales, para comprender la espacialización/materialización de las relaciones sociales, durante el periodo 1892-1942. Este objetivo intentó responderse dentro de un contexto espacio-temporal determinado, analizando la zona denominada Casco Histórico del actual paisaje de la localidad de La Emilia, junto con la zona adyacente de monte, durante el periodo mencionado. El establecimiento del año 1942 como límite se basó en dos criterios fundamentales. Uno interno, delimitado por el cincuentenario de la fábrica-pueblo, comprendiendo el periodo de surgimiento y desarrollo de la fábrica y el crecimiento de la población, junto con la consecuente expansión espacial e incorporación de nuevas estructuras edilicias, que culminó con los grandes festejos a razón de las Bodas de Oro. Otro externo que delimita el momento anterior a la llegada e influencia del peronismo en la comunidad, que supone la introducción de nuevas ideas y cambios en las relaciones sociales y que merece por tanto un abordaje exclusivo.

Junto a este objetivo general, se plantearon una serie de objetivos específicos tendientes a comprender la participación activa del paisaje como producto/productor de un determinado orden social y relaciones de poder a él asociadas: 1) indagando acerca de la incidencia de los dueños de la fábrica en la

1 Escuela de Antropología, Facultad de Humanidades y Artes, Universidad Nacional de Rosario. Entre Ríos 758, 2000, Rosario, SantaFe.d.cadenas@hotmail.com. 
construcción del paisaje para comprender posibles estrategias de control y dominación, así como de resignificación y resistencia, y 2) abordando críticamente el discurso hegemónico de "gran familia" y su papel en la configuración de la vida cotidiana para interpretar significados ocultos bajo la visión oficial.

\section{Aspectos teórico-metodológicos}

Fue necesario, en primera instancia, reflexionar sobre la disciplina misma: qué se considera arqueológico o qué requisitos debe cumplir una determinada problemática para ser considerada arqueológica. En este sentido, fue prioritario re-pasar algunos límites y re-definir algunas definiciones, principalmente al tener en cuenta los criterios tradicionales para determinar si una investigación es arqueológica o si debe enmarcarse bajo otra disciplina, tales como el período temporal analizado, la inclusión de diversos tipos de registros, el abordaje de una materialidad/ espacialidad que se encuentra en uso, la presencia/ausencia de excavaciones en el sentido convencional del término. Este último criterio, puntualmente, parece ser de crucial importancia al momento de catalogar una investigación, por lo que en esta tesis se asumió que no es el acto de excavar lo que define a la disciplina, en tanto técnicas y métodos son elegidos de acuerdo al contexto de investigación y en base a lo que en ese contexto se enfatiza (Hall \& Silliman, 2006). Por el contrario, lo que la disciplina busca es comprender los agentes, las acciones y los significados detrás de la materialidad. Así, si el registro material se encuentra por debajo o sobre la superficie, si el pasado es remoto o reciente (¿hasta dónde llega el pasado?, ¿cuándo empieza el presente?) o si el análisis parte de una taza o una frontera política adquiere poca relevancia. La importancia radica en la información que puedan proporcionar los registros para comprender los sentidos y significados que hay detrás de ellos y construir nuevas narrativas.

Respecto a los lineamientos teóricos en particular, al abordar un paisaje industrial en un contexto histórico, esta tesis podía bien enmarcarse en la Arqueología Histórica, en la Arqueología Industrial, o en la Arqueología del Paisaje. Sin intención de cercarla o limitarla a una única subdisciplina, resultó conveniente reconocer que el caso en cuestión atañe a las tres y que servirse de sus aportes permitiría un mejor abordaje. La arqueología histórica es entendida como el análisis de los restos materiales de la expansión del capitalismo europeo y la consecuente conformación del mundo moderno, que posibilita, a su vez, la inclusión de diversas voces comúnmente marginadas o intencionalmente olvidadas en los relatos escritos, como así también sucesos propios de la vida cotidiana. Por su parte, la Arqueología Industrial es definida como el estudio de la materialidad del proceso de industrialización en la medida en que estableció nuevas formas de organización basadas en nuevas relaciones que dieron lugar al surgimiento de nuevas clases sociales. Establecer, sin embargo, un quiebre entre ambas en el continente americano sería encasillar a la Arqueología Industrial en sí misma, separando a la industria de su contexto amplio y complejo. Es por ello que en esta tesis se considera a la Arqueología Industrial como parte de la Arqueología Histórica en tanto el proceso de industrialización es entendido como un aspecto integrante del proceso de modernización. El paisaje es definido como paisaje industrial, por ser la industria el factor dominante en su conformación y es comprendido como un espacio activo y subjetivo, socialmente producido, colmado de significados y articulado dialécticamente con ciertas prácticas y relaciones sociales (Acuto, 2013; Tilley, 1994).

La metodología implementada se definió de acuerdo al posicionamiento teórico adoptado y al contexto de investigación, de modo que permitiera responder a los objetivos planteados. La tesis se organizó, así, en cinco etapas metodológicas. La primera etapa abordó el primer acercamiento al paisaje a analizar: el "estar" en el campo, la inmersión del cuerpo en él, comprendiendo y vivenciando a través de distintos sentidos y en distintos momentos. Esta interacción con la materialidad/ espacialidad dio lugar a las primeras preguntas disparadoras que activaron un proceso de reflexión e interpretación. Las tres etapas siguientes abordaron cada una por separado un determinado tipo de registro: documentos históricos de diversa índole, registro oral y registro material. Esta división respondió a cuestiones organizativas y no a una jerarquización de las distintas fuentes de información. Por el contrario se reconoció no sólo la importancia de incorporar diversos registros, sino también el valor de cada uno, estableciendo un diálogo entre ellos que permita una mejor comprensión del pasado. Es por ello que la última etapa metodológica consistió en una sistematización final en la que se plantearon puntos claves para relacionar y tensionar lo obtenido en etapas anteriores, y a partir de la cual interpretar los datos en conjunto, analizándolos en sus interrelaciones.

\section{Resultados}

Los objetos pueden adquirir distintos significados y la interpretación puede dar lugar a múltiples lecturas. Sin embargo, en la diversidad de significados se encuentran aquellos que son hegemónicos y que por tanto alcanzan una mayor reproducción y difusión, y logran imponerse por sobre las narrativas y memorias alternativas. Se trata de sentidos oficiales, reconocidos por los actores sociales, que establecen ciertas prácticas y que son impuestos desde las estructuras de poder, en función de sus propios intereses de control y reproducción social (Acuto, 1999, 2013).

Los significados hegemónicos asociados a la materialidad/ espacialidad de La Emilia se expresan y reproducen a través del discurso oficial de la gran familia emiliana. El énfasis puesto en este discurso, y su alcance incluso hasta la actualidad, reproduce las ideas de armonía y cooperación como características propias de este paisaje. El análisis, sin embargo, al poner en discusión distintas fuentes de información permitió encontrar ciertas rupturas y contradicciones que posibilitaron leer más allá de las apariencias y reconstruir La Emilia desde un discurso alternativo.

La Emilia era, en apariencia, un pueblo con una importante fábrica textil. Sin embargo, tanto el registro material como los documentos históricos y los relatos orales permitieron comprender a La Emilia en términos de fábrica-pueblo: paisaje privado cerrado, propiedad de una empresa que es, a su vez, propietaria de todas las estructuras que en él se encuentran, y que en cierto modo lo aísla y protege del exterior, ejerciendo control sobre la espacialidad y sobre quienes la habitan. A su vez, las construcciones, sus características, su tamaño y su disposición establecen marcadas diferencias entre dueños y obreros, y junto con la documentación y reconstrucción de determinadas actividades, eventos y prácticas sociales, son evidencia de una reproducción por fuera de la fábrica de las jerarquías propias del interior de ella, aunque minimizadas en el discurso de gran 
familia. Es por ello que, entiendo que las diferencias de clase y las relaciones de poder, ajustadas a este discurso que les da cierto sentido y al mismo tiempo las disimula, escondiéndolas bajo supuestos lazos íntimos de parentesco que enmascaran vínculos económicos, son muestra del carácter paternalista que impregnó este paisaje.

Reemplazar el concepto de gran familia Emiliana por el de paternalismo de fábrica-pueblo permite enunciar la situación en otros términos, dejando de lado los tintes románticos y pensando en relaciones asimétricas de poder que, apoyadas en el discurso de gran familia, ocultan intereses de clase, persiguiendo claros objetivos. Así mismo, reconocer y visibilizar tales relaciones permitió indagar acerca de posibles resistencias. Si bien el discurso oficial, tan poderoso en este caso, invisibiliza esas resistencias, escondiéndolas bajo montañas de significados hegemónicos, ciertos elementos del paisaje, acciones de los trabajadores y relatos cargados de significaciones vislumbran, aunque de manera tenue, posibles prácticas de resistencia y devuelven la posibilidad de pensar a los habitantes como sujetos activos dentro del entramado de relaciones paternalistas. Esas pequeñas acciones de resistencia analizadas, quizás las más simples que lograron filtrarse entre el ajustado armazón de sentidos oficiales, dejan lugar a nuevas preguntas sobre estas prácticas. Romper con este discurso oficial, enunciándolo ya no como una gran familia, sino como un caso de paternalismo, puede significar el primer paso para encontrar nuevas fisuras, discontinuidades y contradicciones, a través de las que salgan a la luz posibles resistencias y emerjan prácticas independientes de los trabajadores.

Nuevas investigaciones podrían contribuir no sólo a una mejor comprensión sobre este último aspecto en particular, sino también abrir otras líneas de análisis que incorporen nuevas problemáticas y aporten a la construcción crítica del pasado en La Emilia, al mismo tiempo que al desarrollo de una arqueología socialmente significativa.

\section{Bibliografía}

Acuto, F. (1999). Paisaje y dominación: La constitución del espacio social en el Imperio Inka. En A. Zarankin \& F. Acuto (Eds.), Sed Non Satiata. Teoría Social en la Arqueología Latinoamericana Contemporánea, (pp. 33-75). Buenos Aires: Ediciones del Tridente.

Acuto, F. (2013). ¿Demasiados paisajes?: múltiples teorías o múltiples subjetividades en la arqueología del paisaje. Anuario de Arqueología 5, 31-50.

Foucault, M. (2014 [1975]). Vigilar y castigar. Nacimiento de la prisión. Buenos Aires: Siglo Veintiuno Editores.

Foucault, M. (2000[1982]). Space, Knowledge and power. En J. D. Faubion (ed.), Power. The essential Works of Foucault: 1954-1984, Vol. 3. Nueva York: The New York Press.

Hall, M. \& S. Silliman, (2006). Introduction: Archaeology of the modern world. En M. Hall \& S. Silliman (eds.), Historical Archaeology, (pp. 1-19). Oxford: Blackwell Publishing.

McGuire, R. (1991). Edificando el poder en el paisaje cultural del condado de Broome, Nueva York 1880-1940. En R. McGuire \& R. Paynter (Eds.), The Archaeology of Inequality, (pp.
102-124). Cambridge: Blackwell.

Tilley, C. (1994). A phenomenology of landscapes: places, paths and monuments. Oxford: Oxford Berg. 
